\title{
Ovarian, Fallopian Tube, and Primary Peritoneal Carcinoma cM0 TNM Finding v8
}

National Cancer Institute

\section{Source}

National Cancer Institute. Ovarian, Fallopian Tube, and Primary Peritoneal Carcinoma cMO TNM Finding v8. NCI Thesaurus. Code C139930.

Ovarian, fallopian tube, or primary peritoneal carcinoma without evidence of distant metastasis. (from AJCC 8th Ed.) 